\title{
Equilibrium shapes and energies of coherent strained InP islands
}

\author{
Q. K. K. Liu* \\ Bereich Theoretische Physik, Hahn-Meitner-Institut, Glienicker Str. 100, D-14109 Berlin, Germany \\ N. Moll ${ }^{\dagger}$ and M. Scheffler \\ Fritz-Haber-Institut der Max-Planck-Gesellschaft, Faradayweg 4-6, D-14195 Berlin-Dahlem, Germany \\ E. Pehlke \\ Physik-Department T30, Technische Universität München, D-85747 Garching, Germany
}

(Received 14 April 1999)

\begin{abstract}
The equilibrium shapes and energies of coherent strained InP islands grown on GaP have been investigated with a hybrid approach that has been previously applied to InAs islands on GaAs. This combines calculations of the surface energies by density-functional theory and the bulk deformation energies by continuum elasticity theory. The calculated equilibrium shapes for different chemical environments exhibit the $\{101\},\{111\},\{\overline{1} \overline{1} \overline{1}\}$ facets and a (001) top surface. They compare quite well with recent atomic-force microscopy data. Thus in the $\mathrm{InP} / \mathrm{GaInP}$ system a considerable equilibration of the individual islands with respect to their shapes can be achieved. We discuss the implications of our results for the Ostwald ripening of the coherent InP islands. [S0163-1829(99)15147-6]
\end{abstract}

\section{INTRODUCTION}

It was observed about ten years ago that during the heteroepitaxial growth of lattice-mismatched semiconductors small dislocation-free islands can form. ${ }^{1-5}$ To emphasize the zero-dimensional character of their electronic density of states, these objects have been labeled quantum dots. Since then quantum dots have attracted an enormous amount of interest in the area of semiconductor physics. ${ }^{6}$ Besides being fascinating objects for basic research, there are also various potential applications, ranging from improved device properties of quantum-dot semiconductor lasers, which have in fact already been demonstrated, ${ }^{7}$ up to more exotic applications as part of a quantum computer. ${ }^{8}$ The "self-organized" heteroepitaxial growth of arrays of quantum dots with a preferentially narrow size distribution is thus the aim of intense research. To be finally able to optimize the growth parameters, a detailed knowledge of the growth mechanism, its energetics, and its kinetics, is essential.

There is general agreement about the main driving force for, e.g., InAs/GaAs or InP/GaInP island formation in the Stranski-Krastanov growth mode. The growth begins with the deposition of a highly strained two-dimensional film, the wetting layer. With the addition of more material beyond a critical thickness the film becomes metastable. Coherent, i.e., dislocation-free, islands form due to the energy gained by strain relaxation in the islands. However, the details of the growth mechanism ${ }^{9}$ are not yet well understood. In fact, there are competing theories that are based on energy ground-state considerations on the one hand, or on kinetic, i.e., nonequilibrium effects on the other. ${ }^{10-14}$ Accordingly, the final destiny of the islands, as predicted by the various theories, is rather different. The more conventional fate of the islands would be to undergo Ostwald ripening, ${ }^{15,16}$ i.e., the larger islands would grow at the expense of the smaller ones. The resulting island size distribution would be rather broad. In fact, for the systems of concern in our study, Ostwald ripening takes place at a slower rate than the growth rate of the islands. Kinetic effects could be active that effectively decrease the growth rate of the larger islands, thus sharpening the size distribution. ${ }^{11,12,17}$ In contrast to these growth scenarios, it has been suggested ${ }^{18,19}$ that there exists a range of parameters for which islands of a finite size are thermodynamically stable. Our $a b$ initio results for the surface energies and surface stresses, however, indicate that neither in the case of InAs/GaAs nor InP/GaInP would this mechanism result in the formation of stable islands.

For the most widely studied system of InAs islands on GaAs, a range of growth parameters seem to have been established for producing islands of certain uniform densities and a rather narrow size distribution, ${ }^{20-22,6}$ although these growth parameters are still subjects of discussion in the literature. Spectroscopic studies of the islands, after they were capped by barrier materials, have been reported. ${ }^{23-27}$ To investigate the equilibrium shape and stability of uncapped coherent strained InAs islands at low island densities, we have applied a hybrid method to calculate the total energy. ${ }^{28-30} \mathrm{In}$ this approach the energy gained by isolated island formation is decomposed in the following form:

$$
E^{\text {total }}=E^{\text {relax }}+E^{\text {surface }}+E^{\text {edge }}
$$

where $E^{\text {relax }}$ is the gain in deformation energy when the material forms a strained island instead of a biaxially strained film, $E^{\text {surface }}$ is the cost in surface energy due to the creation of facets on the sides of the island instead of the surface covered by the base of the island, and $E^{\text {edge }}$ is the energy cost for the creation of sharp edges. For an isolated island to form at all in preference to a film, $E^{\text {total }}$ must be negative. The surface energy $E^{\text {surface }}$ is calculated $a b$ initio, applying density-functional theory (DFT). In Ref. 29 the surface energies $E^{\text {surface }}$ corresponded to those of unstrained surfaces. 
This approximation was subsequently improved upon in Ref. 30 , in which the renormalization of the surface energies due to surface stress was taken into account. However, the corrections amounted to a reduction of the surface energies by at most $11 \%$ and left the prediction for the equilibrium shape qualitatively unchanged. Furthermore, the edge energy $E^{\text {edge }}$ was estimated to be negligible, provided the island size is not too small. The elastic strain field can be treated within a continuum approach. Thus $E^{\text {relax }}$ is calculated within linear elasticity theory using a finite-element method (FEM). The effect of nonlinearity was seen to be small.

As a result, we obtained a volume-dependent optimum shape for the InAs islands, which can be described as a (001)-truncated pyramid with $\{101\},\{111\}$, and $\{1 \overline{1} 1\}$ faces. However, the diversity of experimentally observed island shapes appears not to be reconcilable with the assumption of thermodynamic equilibrium. Among the experimental results, the mostly square-based islands have faces $\{101\}$, $^{22}$ $\{105\},{ }^{31}\{113\},{ }^{20}\{136\},{ }^{32}$ and a series of islands of low aspect ratios whose morphologies change according to the coverage. ${ }^{21}$ We take this difference as an indication that a sensitivity to growth conditions and kinetic effects have to be featured in a growth theory, including the possibility that the deposited material may migrate or segregate as witnessed in the growth of quantum wells, ${ }^{33,34}$ and self-organized islands. ${ }^{35-37}$

While the predictions of the hybrid method for the equilibrium shape of InAs/GaAs coherent islands have not been borne out so far in experiment, i.e., in the "window" of growth condition assumed, the experimental characterization of the three-dimensional islands in the InP/GaInP system, ${ }^{17}$ briefly summarized in the next paragraph, seems to indicate a better chance for an equilibrium approach to be valid. Therefore, InP/GaInP represents an excellent benchmark system to show both the applicability of the hybrid method and the notion that the shape equilibration of coherent islands can be achieved.

The growth of InP/GaInP by metal-organic vapor phase epitaxy (MOVPE) has consistently yielded uncovered islands ${ }^{17}$ that are significantly larger $\left(\sim 45 \times 60 \mathrm{~nm}^{2}\right)$ than their counterparts in InAs/GaAs $\left(\sim 12 \times 12 \mathrm{~nm}^{2}\right)$ grown by molecular beam epitaxy (MBE). Transmission electron microscopy (TEM) and atomic force microscopy (AFM) have yielded strong evidence that they have facets of low Miller indices only, ${ }^{38,39}$ rather reminiscent of the previous prediction for InAs/GaAs. They are stable against annealing ${ }^{17}$ of several minutes at the growth temperature of $580^{\circ} \mathrm{C}$, and their morphology has been reported to remain unchanged after overgrowth with capping material. ${ }^{40}$ Although for these materials there is a lack of specific observations of segregation inside the islands and diffusion between the islands and the barrier, it is probable that both take place. To what extent they affect the shape and size of InP/GaP islands remains to be determined. It is also becoming clear that the spectroscopy of the islands depends not only on the volume but also significantly on the strain distribution inside the islands, and the latter is greatly influenced by the shape of the islands. ${ }^{41,42}$ The results for InP/GaInP complement those for InAs/GaAs, e.g., growth of quantum wells on InP islands, ${ }^{43}$ growth characteristics, ${ }^{38,44-47}$ photoluminescence, ${ }^{43,44,48-51}$ optical gain and lasing, ${ }^{52} \mathrm{InP}$ islands used as stressors to induce quantum dots in a quantum well, ${ }^{53-57}$ Landau levels formation in InP islands, ${ }^{58}$ and theoretical study of the electronic states of the islands. ${ }^{42,59}$

We are thus encouraged to conduct a study of InP/GaInP islands parallel to our previous work for InAs/GaAs, and hence enlarge our general understanding of coherent strained islands. With the view to comparing our results with experiment $^{38}$ we study uncapped islands of relatively large size, thus ensuring that the approximation of neglecting the edge energy $E^{\text {edge }}$ remains valid. Of course, if the effect of phase segregation inside the island or diffusion of atoms to and from the barrier turns out to be substantial, our approach would need to be generalized. In any event, our findings could still be used as the starting point for a yet more realistic modeling.

The organization of the work is as follows: First, we present the DFT results for the surface energies. In Sec. III, we describe briefly our FEM simulations and derive the equilibrium shape. We shall indicate how Ostwald ripening emerges from our model, assuming that no other faster kinetic processes have preempted its time development. A generalization from InP/GaP to $\mathrm{InP} / \mathrm{Ga}_{x} \mathrm{In}_{1-x} \mathrm{P}$ islands, i.e., to systems with a different lattice mismatch, will be presented.

\section{SURFACE ENERGY}

As in the case of InAs we assume that the relevant surface reconstructions, i.e., those of lowest energies, correspond to the low-index surface orientations $\{110\},\{100\},\{111\}$, and $\{\overline{1} \overline{1} \overline{1}\}$. The details of the calculation of surface energies by DFT are described in Refs. 28 and 30. We apply the local density approximation to the exchange-correlation energy functional and use $a b$ initio norm-conserving, fully separable pseudopotentials. The plane-wave expansion of the wave function has an energy cutoff of $10 \mathrm{Ry}$, and the $\mathbf{k}$ summation makes use of a uniform Monkhorst-Pack mesh with a density equivalent to $64 \mathbf{k}$ points in the complete $(1 \times 1)$ surface Brillouin zone of the (100) surface. We neglect the correction of the surface energies due to strain, as, for the InAs/ GaAs islands, we found it to be small and not affecting our conclusions concerning the island shape and stability. ${ }^{30}$

The surface atomic structures for different surface orientations are shown in Fig. 1. The corresponding surface energies as a function of the phosphorus chemical potential are shown in Fig. 2, where the left and right vertical dashed lines mark the limits for In- and P-rich environments, respectively. They are characterized by the coexistence of the InP surface with either an In or a $\mathrm{P}$ bulk phase. Since epitaxial growth takes place mostly in a P-rich environment, we list in Table I the surface energies of the stable reconstructions for the chemical potential $\mu_{\mathrm{P}}=\mu_{\mathrm{P}}^{\text {bulk }}-0.1 \mathrm{eV}$. It will be shown in Sec. III that the experimentally observed coherent islands are best compared with the theoretical results at this chemical potential.

There are a great deal of similarities between InP and InAs surfaces in equilibrium ${ }^{60,29,30}$ and some interesting differences. Both the (110) and (111) surface energies are independent of the phosphorus chemical potential. The $(1 \times 1)$ relaxed cleavage surface and the stoichiometric In vacancy structure are the stable reconstructions for the (110) and (111) orientations, respectively. The surface energies are 55 

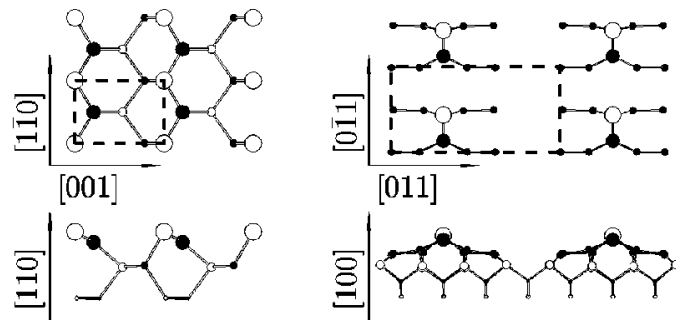

(110) cleavage

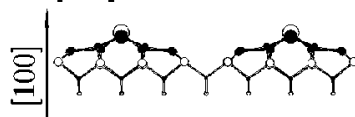

(100) mixed dimer

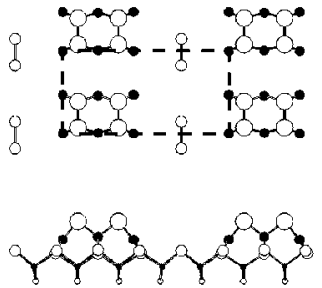

(100) $\beta 2(2 \times 4)$
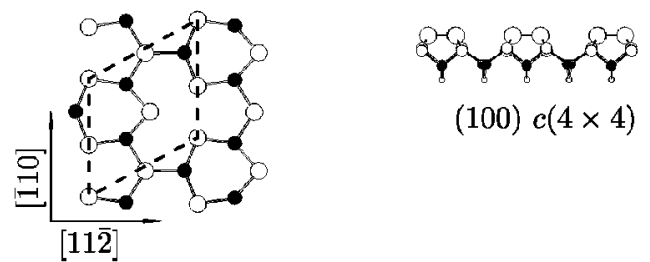

(100) $c(4 \times 4)$
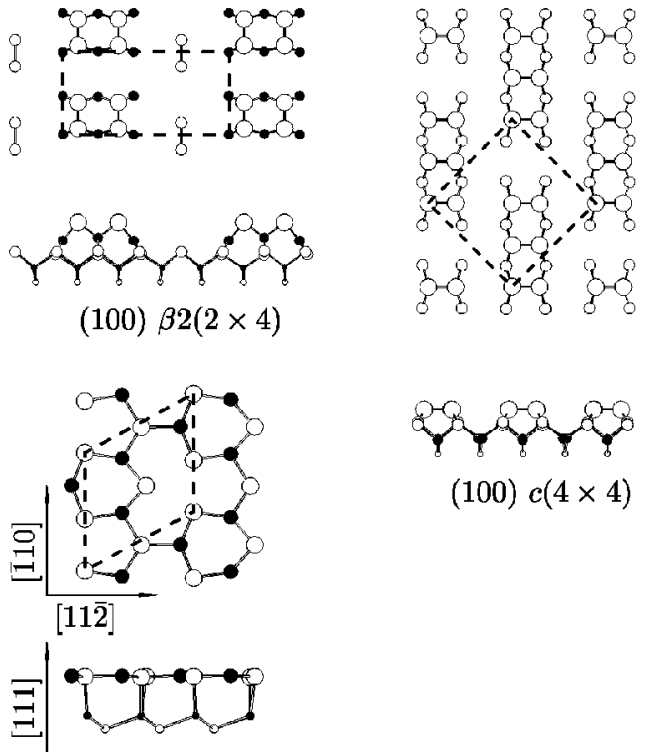

(111) In-vacancy

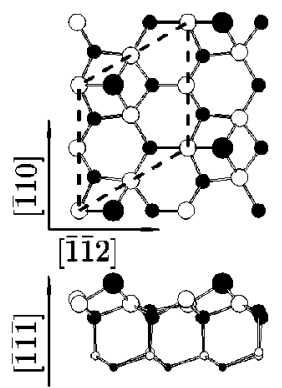

(ī1) In-adatom

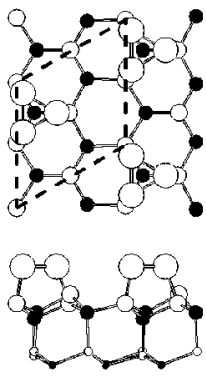

(1̄ī) P-trimer

FIG. 1. Atomic structure models for the different InP surfaces, top and side views. Filled and open circles denote In and $\mathrm{P}$ atoms, respectively.

and $62 \mathrm{meV} / \AA^{2}$. These are to be compared with those of InAs of 41 and $42 \mathrm{meV} / \AA^{2}$. The cleavage surfaces of the (110) orientation in InP have been observed experimentally using low-energy electron diffraction ${ }^{61}$ (LEED) and for both InP and InAs using low-energy positron diffraction. ${ }^{62}$ A DFT study of InP(110) surface has also been carried out. ${ }^{63} \mathrm{We}$ find the equilibrium structure of the ( $\overline{1} \overline{1} \overline{1})$ surfaces to be the same as for InAs. On the ( $\overline{1} \overline{1} \overline{1})$ surface in P-rich environment, the P-trimer reconstruction is preferred, i.e., within some interval near the right-hand dashed line in Fig. 2. At $\mu_{\mathrm{P}}=\mu_{\mathrm{P}}^{\text {bulk }}-0.1 \mathrm{eV}$ the $\operatorname{InP}(\overline{1} \overline{1} \overline{1})$ surface energy is $44 \mathrm{meV} / \AA^{2}$ compared to $36 \mathrm{meV} / \AA^{2}$ for InAs. An In-rich

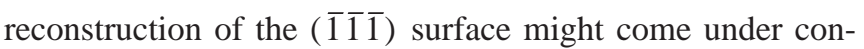
sideration. However, we are not aware of any experimental observation of an In-rich reconstruction. ${ }^{60}$
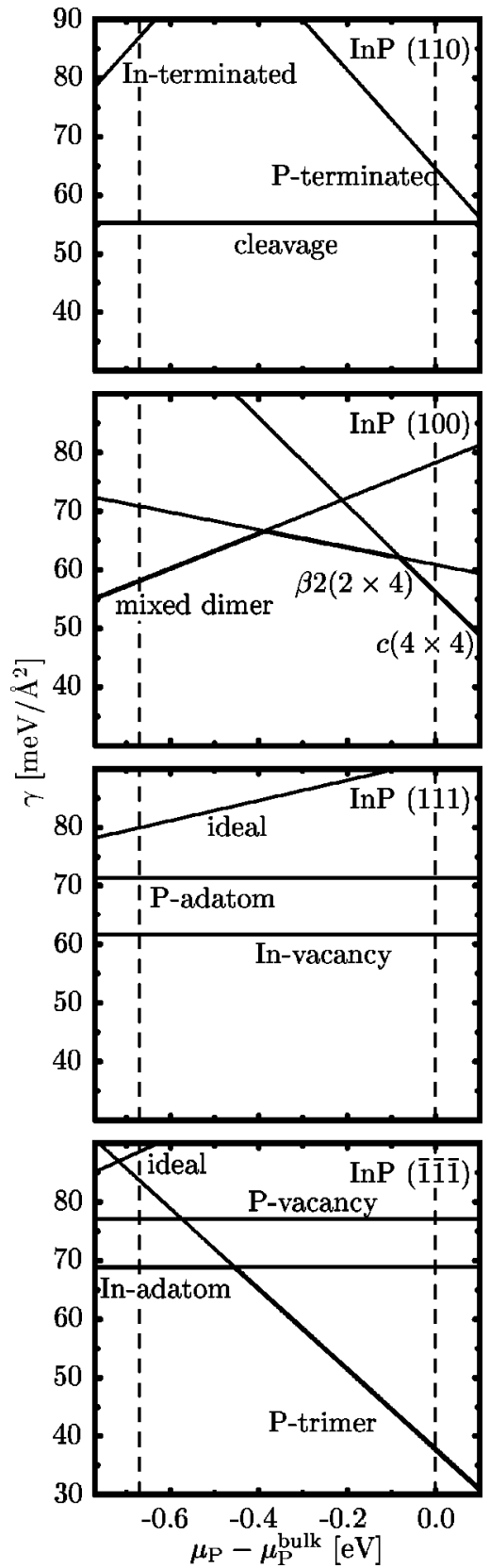

FIG. 2. InP surface energies of the (110), (100), (111), and ( $\overline{1} \overline{1} \overline{1})$ surface orientations as a function of the phosphorous chemical potential. The thick lines highlight the calculated surface energies of the reconstructions of lowest total energy.

The (100) reconstructed surfaces of InP and InAs had been generally assumed to be similar, too. However, recent DFT studies and experimental observations by several groups using low-energy electron diffraction (LEED), reflection anisotropy spectroscopy (RAS), soft x-ray photoelectron spectroscopy (SXPS), and scanning tunneling microscopy (STM) have yielded evidence that there is a qualitative difference between the atomic structure of the $\mathrm{InP}$ and the InAs(100) surfaces. ${ }^{64-66}$ In a very P-rich environment, i.e., at $\mu_{\mathrm{P}}$ rather close to $\mu_{\mathrm{P}}^{\text {bulk }}$, DFT predicts the $c(4 \times 4)$ reconstruction to be more stable than the $\beta 2(2 \times 4)$ reconstruction, which is not the case for InAs. For InP in moderately P-rich and InAs in As-rich environments, $\beta 2(2 \times 4)$ is the common stable reconstruction. Notably, in an In-rich envi- 
TABLE I. The relaxed surface reconstructions of InP for $\mu_{\mathrm{P}}$ $=\mu_{\mathrm{P}}^{\text {bulk }}-0.1 \mathrm{eV}$, and surface energies.

\begin{tabular}{ccc}
\hline \hline Orientation & Reconstruction & $\begin{array}{c}\text { Surface energy } \\
\left(\mathrm{meV} / \AA^{2}\right)\end{array}$ \\
\hline$(110)$ & cleavage & 55 \\
$(100)$ & $\beta 2(2 \times 4)$ & 62 \\
$(111)$ & $(2 \times 2)$ In vacancy & 62 \\
$(\overline{1} \overline{1} \overline{1})$ & $(2 \times 2) \mathrm{P}$ trimer & 44 \\
\hline
\end{tabular}

ronment InP displays a mixed dimer reconstruction, in contrast to the $\alpha(2 \times 4)$ reconstruction for InAs. However, for the growth of strained islands in a moderately P-rich atmosphere, it is still the $\beta 2(2 \times 4)$ reconstruction that is selected as in InAs/GaAs, with the surface energy equal to $62 \mathrm{meV} / \AA^{2}$ compared to $44 \mathrm{meV} / \AA^{2}$ for InAs.

\section{EQUILIBRIUM ISLAND SHAPES}

For a fixed volume of the island (or, equivalently, a fixed number of atoms), the equilibrium shape minimizes the total energy of the system, $E^{\text {total }}$ of Eq. (2), with respect to all possible shapes. As explained above, the surface energy $E^{\text {surface }}$ is calculated for the unstrained surface. The FEM calculations are performed for uncapped islands without a wetting layer as in Refs. 29 and 30. For a square-based island bounded solely by the four $\{101\}$ surfaces, the length of the base is chosen equal to $12.9 \mathrm{~nm}$ and the height to $6.5 \mathrm{~nm}$. This determines the volume that remains unchanged for all calculated islands. The island dimensions vary only moderately for truncated islands bounded by the other low-index surface planes we have included in this study.

We have used the commercial product MARC (Ref. 67) to perform the finite-element simulations. One notable feature of all commercial products is that the preferred finite element (FE) is an eight-node hexahedron or three-dimensional arbitrarily distorted cube. It has been shown that it is superior to the simple tetrahedron in terms of fast convergence and computing speed. We have also adopted this class of FE for all our calculations, with the understanding that corners of the hexahedron can merge to form wedge-shaped or pyramidshaped elements.

We have taken InP to be the island and $\mathrm{GaP}$ to be the substrate material. The corresponding experimental lattice constants and elastic moduli ${ }^{68}$ are listed in Table II. The lattice mismatch is $\alpha:=\left(a_{\mathrm{GaP}}-a_{\mathrm{InP}}\right) / a_{\mathrm{InP}}=-7.1 \%$. Although this mismatch is quite large, we restrict ourselves to linear elasticity theory. Taking the elastic moduli from Table II, the deformation energy per unit volume of the biaxially strained uniform InP film amounts to $3.0 \mathrm{meV} / \AA^{3}$.

The islands are placed $80 \mathrm{~nm}$ apart, and reside on a sub-

TABLE II. The experimental lattice constants $a$ and elastic moduli $c_{11}, c_{12}$, and $c_{44}$ of $\mathrm{InP}$ and $\mathrm{GaP}$.

\begin{tabular}{lcccc}
\hline \hline & $a(\AA)$ & $c_{11}(\mathrm{GPa})$ & $c_{12}(\mathrm{GPa})$ & $c_{44}(\mathrm{GPa})$ \\
\hline $\mathrm{InP}$ & 5.87 & 102 & 58 & 46 \\
$\mathrm{GaP}$ & 5.45 & 141 & 63 & 72 \\
\hline \hline
\end{tabular}

strate of thickness equal to $24 \mathrm{~nm}$. Thus our FEM calculations basically simulate isolated islands. The elastic energy $E^{\text {relax }}$ changes by less than $1 \%$, the adopted accuracy of the FEM calculations, when the island separation is increased by an unit of the island base length. The error induced by the finite thickness of the substrate has been estimated by comparing the elastic energies calculated with two different boundary conditions for the displacement fields at the bottom surface of the slab: the elastic energies calculated for an either fixed or free bottom surface yield an upper and a lower bound to the elastic energy for infinite thickness. ${ }^{69}$ For our choice of the substrate thickness the difference between both bounds is less than $1.5 \%$ of the absolute value of the elastic energy. This accuracy is sufficient for the comparison of the various island shapes focused on in this work (note, however, that thicker slabs would be necessary to calculate accurately the interaction energy between the islands). In the simulation, the island and substrate surfaces are not subjected to any boundary conditions, while periodic boundary conditions are applied to the displacement field on the side planes of the FE cell. In our FEM calculations of $E^{\text {relax }}$ the number of FE varies slightly, depending on the island's bounding surfaces. The number of $\mathrm{FE}$ is increased in the areas where the deformation energy density is large until a $1 \%$ accuracy is achieved. In general, there are approximately $7000 \mathrm{FE}$ in total, of which approximately 2000 are distributed in the island. For volume-conserving truncated islands, the elastic energy can be approximated with sufficient accuracy from the untruncated value in a simple way. ${ }^{29}$ The idea behind this approximation is that truncating the relaxed top portion of an uncapped island should leave the $E^{\text {relax }}$ of the island nearly unchanged and the remaining volume is renormalized to maintain volume conservation. The approximation is more accurate the less strained material is contained in the truncated portion. In our present study, we concentrate on truncated islands that do not violate this approximation.

The anisotropy of the surface energy driving the formation of particular crystal facets is an essential aspect of our approach. ${ }^{70}$ We have investigated island shapes which are bounded by the low-Miller-index surfaces described in Sec. II. The collection of shapes we have considered is the same as in the study of the InAs islands. ${ }^{29,30}$ The island base has the orientation (001) and the top of the island may be truncated by a plane of the same orientation (see Fig. 6 of Ref. 30).

The results of energy evaluated according to Eq. (2) are summarized in a scale-invariant manner (for isolated islands) in Fig. 3, where the ordinate $x=E^{\text {surface }} / V^{2 / 3}$ and abscissa $y$ $=E^{\text {relax }} / V$. We have taken the surface energies at the phosphorus chemical potential $\mu_{\mathrm{P}}=\mu_{\mathrm{P}}^{\text {bulk }}-0.1 \mathrm{eV}$. The solid symbols denote untruncated islands for which full FE calculations have been carried out. Starting with a data point of a peaked island, as the relaxed top portion of the island is incrementally sliced off and the size of the remaining island is increased to maintain volume conservation, the strain energy of the island increases. This is directly related to the increase of $y=E^{\mathrm{relax}} / V$ in Fig. 3. Hence the data points move towards the top left corner of Fig. 3 as the islands become flatter. Qualitatively these results are similar to those of InAs/GaAs. ${ }^{29,30}$ We find that a square-based island with $\{101\}$ facets only (solid square in Fig. 3) has a larger bulk 


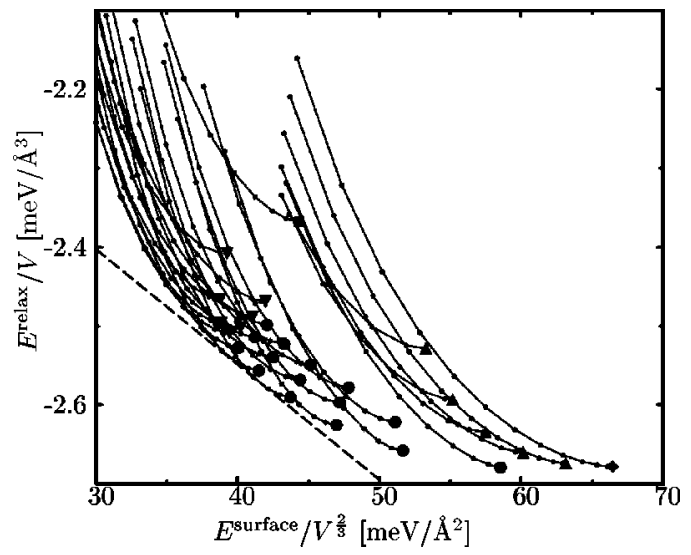

FIG. 3. The relaxation energy per unit volume $E^{\text {relax }} / V$ versus $E^{\text {surface }} / V^{2 / 3}$ for InP islands. Square: square-based island with four $\{101\}$ facets. Diamond: square-based island with two $\{111\}$ and two $\{\overline{1} \overline{1}\}$ facets. Triangles up: huts with two $\{111\}$ and two $\{\overline{1} \overline{1} \overline{1}\}$ facets. Triangles down: square-based $\{101\}$ island with $\{\overline{1} \overline{1} \overline{1}\}$ truncated edges. Dots: islands with four $\{101\}$, two $\{111\}$, and two $\{\overline{1} \overline{1} \overline{1}\}$ facets. The dashed line is the line of constant total energy $E^{\text {relax }}+E^{\text {surface }}=$ const that selects the equilibrium shape for the volume $V=4 \times 10^{5} \AA^{3}$.

deformation energy than a square-based island with two $\{111\}$ and two $\{\overline{1} \overline{1} \overline{1}\}$ facets (rhombus). The latter has steeper side facets which allow a more efficient stress relaxation. The line that emanates from each of the solid symbols joins up the small dots for which $E^{\text {relax }}$ was derived from the analytical approximation for the volume-conserving truncated island. For a given volume $V$, islands with the same total energy $E^{\text {total }}$ lie on the straight line

$$
\frac{E^{\text {total }}}{V}=\frac{E^{\text {relax }}}{V}+\frac{E^{\text {surface }}}{V}=y+V^{-1 / 3} x=\text { const, }
$$

plotted as a dashed line in Fig. 3. (Note that the edge energies $E^{\text {edge }}$ have been neglected.) It is clear from Eq. (2) that the volume of the island $V$ is related to the negative slope of the line. For a given volume $V$, the equilibrium shape of the island is determined by the first point of contact from below of the straight line, Eq. (2), with the calculated island-energy curves.

For a given island shape, $E^{\text {relax }}$ and $E^{\text {surface }}$ scale like $V$ and $V^{2 / 3}$, respectively. This means that the data in Fig. 3 are invariant against a change of the volume of all islands by the same factor. However, the slope of the straight line, Eq. (2), changes, and hence a different equilibrium shape will be selected. By inspection, the results of Fig. 3 indicate that for larger $V$, i.e., smaller negative slope, untruncated islands are most likely to be selected, while smaller $V$ favors truncated islands as equilibrium shape. The interplay of $E^{\text {relax }}$ and $E^{\text {surface }}$ in Eq. (2), and how their relative weightings change as a function of the island volume have been discussed and supported by explicit calculations in Ref. 29. Figure 3 serves as a compact way of encapsulating the result for the equilibrium shape of a noninteracting island of arbitrary volume, provided that the volume (or the number of atoms) of the island is not too small to invalidate our hybrid ansatz, Eq. (2), or too large such that the island is no longer coherent
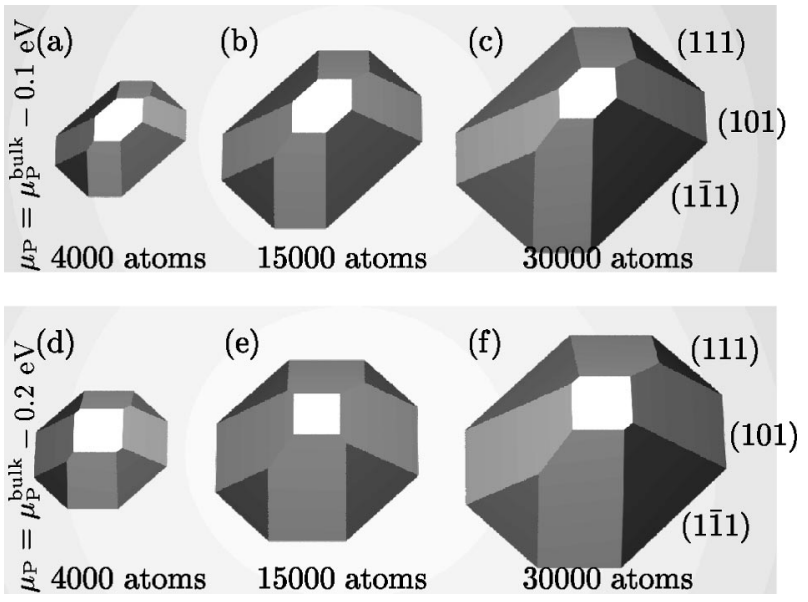

FIG. 4. The equilibrium shapes of coherent strained InP islands for three different volumes and two different chemical potentials. The unstrained volumes in ascending order are approximately $1 \times 10^{5} \AA^{3}, 4 \times 10^{5} \AA^{3}, 8 \times 10^{5} \AA^{3}$.

(dislocations appear). One should note in addition the implied assumption that as larger islands are selected, the islands remain noninteracting.

We display in Fig. 4 examples of equilibrium shapes for three different island volumes. They illustrate nicely that for a smaller volume a relatively larger amount of material is truncated from the top of the island. Additionally, we can also inspect the difference in shapes due to a variation of the chemical potential: The $\{1 \overline{1} 1\}$ facet, which is favored in a P-rich environment, is consistently more prominent for $\mu_{\mathrm{P}}$ $=\mu_{\mathrm{P}}^{\text {bulk }}-0.1 \mathrm{eV}$ than for $\mu_{\mathrm{P}}=\mu_{\mathrm{P}}^{\text {bulk }}-0.2 \mathrm{eV}$. The equilibrium shapes shown in Fig. 4 have many similarities with the experimentally observed islands, ${ }^{38,39}$ in particular all observed facets are well accounted for by our theory. Note that the experiments have been carried out for InP/GaInP instead of InP/GaP; thus the experimental and theoretical lattice mismatch differ by nearly a factor of two. However, this can be taken care of by a simple rescaling of the island volumes.

The experimental data on the growth of InP islands mostly involve InP grown on GaInP lattice-matched to a GaAs substrate. ${ }^{38,39,44-52}$ In the cases where InP islands were used as stressors of quantum wells, they were grown on GaAs. In all these cases the lattice-mismatch amounts to about $-3.7 \%$. TEM and AFM have been used to characterize the coherent strained InP islands and their lateral sizes are usually found to be roughly $40 \mathrm{~nm} \times 60 \mathrm{~nm}$ and the height between $15 \mathrm{~nm}$ and $20 \mathrm{~nm}$. In our study we have assumed a $\mathrm{GaP}$ substrate with a lattice mismatch of $-7.1 \%$. Therefore, our data have to be rescaled before comparing our equilibrium shapes with the experimental observations. The equilibrium shape at a given volume $V$ and lattice mismatch $\alpha$ is calculated by minimizing the total energy $E^{\text {total }}(\alpha, V, I)$ with respect to the island shape $I$. As above, we omit the contribution from the edge energy and the renormalization of the surface energy due to the strain, and make use of the scaling behavior of the elastic energy $E^{\text {relax }}$ and surface energy $E^{\text {surface }}$

$$
E^{\text {relax }}(\alpha, V, I)=E_{0}^{\text {relax }}(I) \alpha^{2} V
$$




$$
E^{\text {surface }}(V, I)=E_{0}^{\text {surface }}(I) V^{2 / 3},
$$

where $E_{0}^{\text {relax }}(I)$ and $E_{0}^{\text {surface }}(I)$ are constants of proportionality that depend only on the shape. The total energy of the equilibrium island follows from

$$
E^{\text {total }}(\alpha, V)=\alpha^{2} V \min _{I}\left[E_{0}^{\text {relax }}(I)+E_{0}^{\text {surface }}(I) \alpha^{-2} V^{-1 / 3}\right] .
$$

Therefore the optimum island shape is the same for volumes $V, V^{\prime}$ and lattice mismatches $\alpha, \alpha^{\prime}$, respectively, provided that the relation

$$
\alpha^{2} V^{1 / 3}=\alpha^{\prime 2} V^{1 / 3}
$$

holds. This simple relation will be altered, however, if the different elastic moduli for $\mathrm{GaP}$ and $\mathrm{GaInP}$ are taken into account. According to Eq. (6), for a reduced lattice mismatch of $\alpha^{\prime}=-3.7 \%$, the equilibrium island shapes displayed in Fig. 4 correspond to the enlarged volumes $V^{\prime}=5$ $\times 10^{6} \AA^{3}, 2 \times 10^{7} \AA^{3}$, and $4 \times 10^{7} \AA^{3}$. The volume of the islands observed in Refs. 38 and 39 ranges from 9 $\times 10^{6} \AA^{3}$ to $2 \times 10^{7} \AA^{3}$. In view of all the uncertainties, the agreement between the observed and calculated island shapes and volumes are quite reasonable. Note that the interdiffusion of In and Ga from the InP island and GaInP substrate would decrease the assumed lattice mismatch of $-3.7 \%$, hence further increasing the scaled volumes according to Eq. (6), and would also alter the effective elastic moduli of the island and barrier materials. Although this topic has yet to be explored in these materials, there is clear evidence $^{71,72}$ for In and $\mathrm{Ga}$ interdiffusion in the growth of $\mathrm{In}_{x} \mathrm{Ga}_{1-x}$ As islands embedded in GaAs.

Finally we briefly turn to the question of the long-timescale evolution of an ensemble of islands. In the theory of Shchukin et al., ${ }^{18}$ a combination of material parameters determines whether thermodynamic equilibration drives the system towards a stable array of islands or whether Ostwald ripening occurs. A stable array would arise, if $E^{\text {surface }}$ in Eq. (2) is modified by the surface strain in such a way that it changes sign. Making use of our quantitative surface energies, we can estimate, whether this condition is likely to be fulfilled or not in the case of InP.

The change in surface energy $E^{\text {surface }}$ is given by a sum over all facets of the island:

$$
E^{\text {surface }}=\sum_{i}^{\text {facets }} \gamma^{i} A^{i}-\gamma^{\text {base }} A^{\text {base }}=A^{\text {base }} \sum_{i}^{\text {facets }}\left[\frac{\gamma^{i}}{\cos \theta^{i}}-\gamma^{\text {base }}\right] \nu^{i},
$$

where $A^{\text {base }}$ and $A^{i}$ denote the areas of the base and the $i$ th facet on the surface of the island, respectively, $\theta^{i}$ is the angle between the $i$ th facet and the substrate surface, and $\nu^{i}$ is the ratio of $A^{i}$ projected onto the base and $A^{\text {base }}$. The $\gamma^{i}$ are the surface energies from Table I. Each term within the summation of Eq. (7) is positive, and hence $E^{\text {surface }}$ is positive. This has the consequence that, for a fixed equilibrium island shape, the volume derivative of Eq. (2) is always negative, i.e., the total energy per unit volume decreases as the volume increases, thus favoring larger and larger islands, leading to Ostwald ripening. To change the sign of the respective individual contribution to Eq. (7), a strain-induced renormaliza- tion of the surface energy of approximately $20 \%$ for the facets with (101) and ( $\overline{1} \overline{1} \overline{1})$ orientations, and $40 \%$ for the facet with (111) orientation would be necessary. For the case of InAs/GaAs, the renormalization amounted to at most 11 $\% .^{30,73}$ Therefore, it seems reasonable to argue that Ostwald ripening is also the fate of the InP islands. Furthermore, a majority of the InP islands are grown on GaInP substrates whose lattice mismatch is about $50 \%$ smaller than in the InP/GaP system. This further diminishes the importance of the renormalization of the surface energy due to the strain field. Altogether this discussion seems to indicate that we are outside the parameter range for which arrays of stable islands occur. It is worth noting that the parameter range that conforms to Shchukin et al. ${ }^{18}$ implies that $E^{\text {surface }}$ in Eq. (2) changes sign due to stress, i.e., one has the unusual situation that the total energy is lowered by creating new surfaces. Furthermore, the Shchukin et al. model includes edge energies and island-island interaction to prevent the islands from coalescing. In comparison, the approach of Ref. 74 to tackle the islands has a much less restrictive ansatz and the agreement with experiments is encouraging.

\section{SUMMARY AND CONCLUSION}

We have applied a hybrid method to calculate the equilibrium shape of large uncapped isolated coherent strained islands of InP on a GaInP substrate. Our theoretical results for InP/GaInP have much in common with our previous findings for InAs/GaAs, while the experimentally observed islands for the two different material combinations look quite different. The dominance of the $\{\overline{1} \overline{1} \overline{1}\}$ InP facets relative to the $\{101\}$ and $\{111\}$ facets in a P-rich atmosphere is analogous to the large $\{\overline{1} \overline{1} \overline{1}\}$ InAs facets occurring under As-rich conditions. $^{29}$ For InP the dominance is even more pronounced, which leads to a noticeable difference between the equilibrium island shapes. In the case of InAs the modification of the surface energies by surface stress enhances this dominance further. ${ }^{30}$ One could expect this to be true also for InP, although explicit calculations of the surface stress have not been carried out.

Our results for InP/GaInP are in qualitative agreement with experiments, in that all the observed facets are accounted for by our theory. Quantitatively, the width-tolength ratio of 1.4 is well reproduced. We take this as an encouraging evidence that the coherent InP islands most often observed in experiments are close to local thermodynamic equilibrium. Moreover, this agreement further corroborates the validity of our theoretical approach, i.e., if it is the equilibrium shape we are interested in, our method provides a reliable answer-and this should also be true for InAs/GaAs.

However, the experimental islands shapes do not agree with the equilibrium island shapes for InAs/GaAs as we find for InP/GaInP. In view of the distinct variety of observed InAs island shapes, this discrepancy points towards the importance of kinetic effects as the missing ingredient of a more comprehensive theory for those islands. This ought to include a description of interdiffusion as exemplified by empirical evidence. ${ }^{71,72}$

We find that the contribution of the surface energies to the total energy of an InP island is positive, and it seems un- 
likely that the sign of this contribution would change by surface stress. Thus our results favor Ostwald ripening of the islands (as opposed to the creation of a thermodynamically stable array of islands). However, for fixed nucleation points, i.e., island density, the island volume is driven by the equilibrium of the islands and the wetting layer. ${ }^{74}$

Finally, we briefly note that our approach can be applied to other materials. There has been recent report of selfassembled $\mathrm{GaN}$ islands of base diameter $17 \mathrm{~nm}$ and height 4

*Electronic address: liu@hmi.de

†Present address: Dept. of Physics, Massachusetts Institute of Technology, Cambridge, MA 02139.

†Present address: Universität Essen, FB Physik, Universitätsstraße 2, D-45117 Essen, Germany.

${ }^{1}$ D. J. Eaglesham and M. Cerullo, Phys. Rev. Lett. 64, 1943 (1990).

${ }^{2}$ Y.-W. Mo, D. E. Savage, B. S. Swartzentruber, and M. G. Lagally, Phys. Rev. Lett. 65, 1020 (1990).

${ }^{3}$ S. Guha, A. Madhukar, and K. C. Rajkumar, Appl. Phys. Lett. 57, 2110 (1990).

${ }^{4}$ C. W. Snyder, B. G. Orr, D. Kessler, and L. M. Sander, Phys. Rev. Lett. 66, 3032 (1991).

${ }^{5}$ B. G. Orr, D. Kessler, C. W. Snyder, and L. M. Sander, Europhys. Lett. 19, 33 (1992).

${ }^{6}$ D. Bimberg, M. Grundmann, and N. N. Ledentsov, Quantum Dot Heterostructures (Wiley, Chichester, UK, 1998).

${ }^{7}$ N. Kirstaedter, N. N. Ledentsov, M. Grundmann, D. Bimberg, V. M. Ustinov, S. S. Ruvimov, M. V. Maximov, P. S. Kop'ev, Z. I. Alferov, U. Richter, P. Werner, U. Gösele, and J. Heydenreich, Electron. Lett. 30, 1416 (1994).

${ }^{8}$ P. Zanardi and F. Rossi, Phys. Rev. Lett. 81, 4752 (1998).

${ }^{9}$ R. Heitz, T. R. Ramachandran, A. Kalburge, Q. Xie, I. Mukhametzhanov, P. Chen, and A. Madhukar, Phys. Rev. Lett. 78, 4071 (1997).

${ }^{10}$ C. Priester and M. Lannoo, Phys. Rev. Lett. 75, 93 (1995).

${ }^{11}$ Y. Chen and J. Washburn, Phys. Rev. Lett. 77, 4046 (1996).

${ }^{12}$ D. E. Jesson, K. M. Chen, and S. J. Pennycock, Mater. Res. Bull. 21, 31 (1996); D. E. Jesson, G. Chen, K. M. Chen, and S. J. Pennycock, Phys. Rev. Lett. 80, 5156 (1998).

${ }^{13}$ H. T. Dobbs, D. D. Vvedensky, A. Zangwill, J. Johansson, N. Carlsson, and W. Seifert, Phys. Rev. Lett. 79, 897 (1997).

${ }^{14}$ J. Tersoff, Phys. Rev. Lett. 81, 3183 (1998).

${ }^{15}$ W. Ostwald, Z. Phys. Chem., Stoechiom. Verwandtschaftsl. 34, 495 (1900).

${ }^{16}$ M. Zinke-Allmang, L. C. Feldman, and M. H. Grabow, Surf. Sci. Rep. 16, 377 (1992)

${ }^{17}$ W. Seifert, N. Carlsson, M. Miller, M.-E. Pistol, L. Samuelson, and L. R. Wallenberg, Prog. Cryst. Growth Charact. Mater. 33, 423 (1996).

${ }^{18}$ V. A. Shchukin, N. N. Ledentsov, P. S. Kop'ev, and D. Bimberg, Phys. Rev. Lett. 75, 2968 (1995)

${ }^{19}$ I. Daruka and A.-L. Barabási, Phys. Rev. Lett. 79, 3708 (1997).

${ }^{20}$ J. M. Moison, F. Houzay, F. Barthe, L. Leprince, E. André, and O. Vatel, Appl. Phys. Lett. 64, 196 (1994).

${ }^{21}$ D. Leonard, K. Pond, and P. M. Petroff, Phys. Rev. B 50, 11687 (1994).

${ }^{22}$ S. Ruvimov, P. Werner, K. Scheerschmidt, U. Gösele, J. Heydenreich, U. Richter, N. N. Ledentsov, M. Grundmann, D. Bimberg, V. M. Ustinov, A. Y. Egorov, P. S. Kop'ev, and Z. I. Alferov, nm grown on AlN substrate and capped by AlN. ${ }^{75}$ This wide band-gap material is at present intensely studied because of its capability in blue light emission.

\section{ACKNOWLEDGMENTS}

This work was supported in part by the Sfb 296 of the Deutsche Forschungsgemeinschaft.

Phys. Rev. B 51, 14766 (1995)

${ }^{23}$ M. Grundmann, J. Christen, N. N. Ledentsov, J. Böhrer, D. Bimberg, S. Ruvimov, P. Werner, U. Richter, U. Gösele, J. Heydenreich, V. M. Ustinov, A. Y. Egorov, A. E. Zhukov, P. S. Kop'ev, and Z. I. Alferov, Phys. Rev. Lett. 74, 4043 (1995).

${ }^{24}$ K. H. Schmidt, G. Medeiros-Ribeiro, M. Oestreich, and P. M. Petroff, Phys. Rev. B 54, 11346 (1996).

${ }^{25}$ R. Heitz, M. Grundmann, N. N. Ledentsov, L. Eckey, M. Veit, D. Bimberg, V. M. Ustinov, A. Y. Egorov, A. E. Zhukov, P. Kop'ev, and Z. Alferov, Appl. Phys. Lett. 68, 361 (1996).

${ }^{26}$ S. Sauvage, P. Boucaud, F. H. Julien, J.-M. Gérard, and J.-M. Marzin, J. Appl. Phys. 82, 3396 (1997).

${ }^{27}$ B. T. Miller, W. Hansen, S. Manus, R. J. Luyken, A. Lorke, J. P. Kotthaus, S. Huant, G. Medeiros-Ribeiro, and P. M. Petroff, Phys. Rev. B 56, 6764 (1997).

${ }^{28}$ N. Moll, A. Kley, E. Pehlke, and M. Scheffler, Phys. Rev. B 54, 8844 (1996).

${ }^{29}$ E. Pehlke, N. Moll, A. Kley, and M. Scheffler, Appl. Phys. A: Mater. Sci. Process. 65, 525 (1997).

${ }^{30}$ N. Moll, M. Scheffler, and E. Pehlke, Phys. Rev. B 58, 4566 (1998).

${ }^{31}$ G. S. Solomon, M. C. Carlson, and J. S. Harris, Appl. Phys. Lett. 69, 1897 (1996).

${ }^{32}$ H. Lee, R. Lowe-Webb, W. Yang, and P. Sercel, J. Appl. Phys. 72, 812 (1998).

${ }^{33}$ K. Muraki, S. Fukatsu, Y. Shiraki, and R. Ito, Appl. Phys. Lett. 61, 557 (1992)

${ }^{34}$ J. Leymarie, C. Monier, A. Vasson, A.-M. Vasson, M. Leroux, B. Courboulés, N. Grandjean, C. Deparis, and J. Massies, Phys. Rev. B 51, 13274 (1995).

${ }^{35}$ Qianghua Xie, P. Chen, and A. Madhukar, Appl. Phys. Lett. 65, 2051 (1994).

${ }^{36}$ N. Grandjean, J. Massies, and O. Tottereau, Phys. Rev. B 55, R10 189 (1997).

${ }^{37}$ J. M. Garcia, G. Medeiros-Ribeiro, K. Schmidt, T. Ngo, J. L. Peng, A. Lorke, J. Kotthaus, and P. M. Petroff, Appl. Phys. Lett. 71, 2014 (1997).

${ }^{38}$ K. Georgsson, N. Carlsson, L. Samuelson, W. Seifert, and L. R. Wallenberg, Appl. Phys. Lett. 67, 2981 (1995).

${ }^{39}$ M.-E. Pistol, J.-O. Bovin, A. Carlsson, N. Carlsson, P. Castrillo, K. Georgsson, D. Hessman, T. Junno, L. Montelius, C. Persson, L. Samuelson, W. Seifert, and L. R. Wallenberg, in Proceedings of the 23rd International Conference on the Physics of Semiconductors, edited by M. Scheffler and R. Zimmermann (World Scientific, Singapore, 1996), p.1317.

${ }^{40}$ A. Carlsson, L. R. Wallenberg, C. Persson, and W. Seifert, Surf. Sci. 406, 48 (1998).

${ }^{41}$ J. Kim, L.-W. Wang, and A. Zunger, Phys. Rev. B 57, R9408 (1998). 
${ }^{42}$ C. Pryor, M.-E. Pistol, and L. Samuelson, Phys. Rev. B 56, 10404 (1997).

${ }^{43}$ J. Ahopelto, H. Lipsanen, M. Sopanen, T. Koljonen, and H. E.-M. Niemi, Appl. Phys. Lett. 65, 1662 (1994).

${ }^{44}$ A. Kurtenbach, K. Eberl, and T. Shitara, Appl. Phys. Lett. 66, 361 (1995).

${ }^{45}$ C. M. Reaves, V. Bressler-Hill, S. Varma, W. H. Weinberg, and S. P. DenBaars, Surf. Sci. 326, 209 (1995).

${ }^{46}$ C. M. Reaves, R. I. Pelzel, G. C. Hsueh, W. H. Weinberg, and S. P. DenBaars, Appl. Phys. Lett. 69, 3878 (1996).

${ }^{47}$ B. Junno, T. Junno, M. S. Miller, and L. Samuelson, Appl. Phys. Lett. 72, 954 (1998)

${ }^{48}$ M.-E. Pistol, N. Carlsson, C. Persson, W. Seifert, and L. Samuelson, Appl. Phys. Lett. 67, 1438 (1995).

${ }^{49}$ P. Castrillo, D. Hessman, M.-E. Pistol, S. Anand, N. Carlsson, W. Seifert, and L. Samuelson, Appl. Phys. Lett. 67, 1905 (1995).

${ }^{50}$ S. Anand, N. Carlsson, M.-E. Pistol, L. Samuelson, and W. Seifert, Appl. Phys. Lett. 67, 3016 (1995).

${ }^{51}$ D. Hessman, P. Castrillo, M.-E. Pistol, C. Pryor, and L. Samuelson, Appl. Phys. Lett. 69, 749 (1996).

${ }^{52}$ A. Moritz, R. Wirth, A. Hangleiter, A. Kurtenbach, and K. Eberl, Appl. Phys. Lett. 69, 212 (1996).

53 M. Sopanen, H. Lipsanen, and J. Ahopelto, Appl. Phys. Lett. 66, 2364 (1995).

${ }^{54}$ J. Tulkki and A. Heinämäki, Phys. Rev. B 52, 8239 (1995).

${ }^{55}$ M. Sopanen, M. Taskinen, H. Lipsanen, and J. Ahopelto, Appl. Phys. Lett. 69, 3393 (1996).

${ }^{56}$ H. Lipsanen, M. Sopanen, and J. Ahopelto, Phys. Rev. B 51, 13868 (1995).

${ }^{57}$ S. Grosse, J. H. H. Sandmann, G. von Plessen, J. Feldmann, H. Lipsanen, M. Sopanen, J. Tulkki, and J. Ahopelto, Phys. Rev. B 55, 4473 (1997).

${ }^{58}$ S. Nomura, L. Samuelson, M.-E. Pistol, K. Uchida, N. Miura, T. Sugano, and Y. Aoyagi, Appl. Phys. Lett. 71, 2316 (1997).

${ }^{59}$ H. Fu and A. Zunger, Phys. Rev. B 55, 1642 (1997); 56, 1496 (1997)

${ }^{60}$ W. Mönch, Semiconductor Surfaces and Interfaces (SpringerVerlag, Berlin, 1993).

${ }^{61}$ W. K. Ford, T. Guo, K. J. Wan, and C. B. Duke, Phys. Rev. B 45,
11896 (1992).

${ }^{62}$ X. M. Chen, K. F. Cantor, C. B. Duke, A. Paton, D. L. Lessor, and W. K. Ford, Phys. Rev. B 48, 2400 (1993).

${ }^{63}$ A. Umerski and G. P. Srivastava, Surf. Sci. 307-309, 680 (1994).

${ }^{64}$ J. Kinsky, Ch. Schultz, D. Pahlke, A. M. Frisch, T. Hermann, M. Esser, and W. Richter, Appl. Surf. Sci. 123/124, 228 (1998).

${ }^{65}$ P. J. Parbrook, K. B. Ozanyan, M. Hopkinson, C. R. Whitehouse, Z. Sobiersierski, and D. I. Westwood, Appl. Surf. Sci. 123/124, 313 (1998).

${ }^{66}$ W. G. Schmidt, F. Bechstedt, N. Esser, M. Pristovsek, Ch. Schultz, and W. Richter, Phys. Rev. B 57, 14596 (1998).

${ }^{67}$ MARC Analysis Research Corp., User's Guide 1996, Palo Alto, CA.

${ }^{68}$ Landolt-Börnstein, New Series III/11, edited by K.-H. Hellwege and A. M. Hellwege (Springer-Verlag, Berlin, 1979), p. 27; ibid., New Series III/17a (Springer-Verlag, Berlin, 1982), pp. 204, 287.

${ }^{69}$ This follows from the variational property of the elastic energy with respect to the displacement field. Deep inside a semiinfinite slab the displacement field goes to zero. When a plane on which the displacement is forced to be zero is artificially placed at some finite depth, the elastic energy increases. However, when the lower portion of the semi-infinite slab up to the same finite depth is discarded and the slab is allowed to relax, the elastic energy decreases. These are the upper and lower bounds of the elastic energy for the chosen thickness of the slab.

${ }^{70}$ B. J. Spencer and J. Tersoff, Phys. Rev. Lett. 79, 4858 (1997).

${ }^{71}$ U. Woggon, W. Langbein, J. M. Hvam, A. Rosenauer, T. Remmele, and D. Gerthsen, Appl. Phys. Lett. 71, 377 (1997).

${ }^{72}$ A. Rosenauer, T. Remmele, D. Gerthsen, K. Tillmann, and A. Förster, Optik (Stuttgart) 105, 99 (1997).

${ }^{73}$ N. Moll, Theorie der Form und Stabilität von Quantenpunkten auf III-V-Halbleitern (Wissenschaft und Technik Verlag, Berlin, 1998).

${ }^{74}$ L. G. Wang, P. Kratzer, M. Scheffler, and N. Moll, Phys. Rev. Lett. 82, 4042 (1999).

${ }^{75}$ F. Widmann, J. Simon, B. Daudin, G. Fouillet, J. L. Rouviere, N. T. Pelekanos, and G. Fishman, Phys. Rev. B 58, R15 989 (1998). 